\title{
Konversion zum Islam als Implementation von Geschlechtsehre ${ }^{1}$
}

\section{Monika Wohlrab-Sahr}

FU Berlin, Institut für Bildungssoziologie, Arnimallee 11, D-14195 Berlin

Zusammenfassung: Der Text befaßt sich auf Grundlage einer empirischen Studie mit der Konversion deutscher Männer und Frauen zum Islam. Gewählt wird dabei ein funktionaler Zugang: es geht darum, das biographische Problem zu rekonstruieren, für das die Konversion eine Lösung darstellt. Anhand von drei Fällen wird ein bestimmter Typus der Konversion zum Islam herausgearbeitet, bei dem diese die Funktion erfüllt, das Problem der Verletzung von Sexualnormen und damit verbundener sozialer Entwertung zu bewältigen. Diese Bewältigung geschieht auf dem Wege symbolischer Transformation, durch die biographische Erfahrungen einerseits „präsentiert“ werden, gleichzeitig aber durch neue Rahmungen oder durch Verschiebungen in den religiösen Sinnbereich ihre konkrete, personal verankerte lebensgeschichtliche Problematik verlieren. Der Islam wird von den Repräsentanten dieses Typs als „Religion der Moral“ perzipiert, dem sie Ehrkodizes und auf Geschlechtsidentität zielende präsentative Symbole entlehnen. Diese Symboliken werden so von außen in ein kulturelles System implementiert, zu dessen sozial verbindlichem Inventar sie nicht mehr gehören, das aber dennoch Phänomene sozialer Entwertung produziert.

\section{Einleitung}

Mit der gestiegenen Aufmerksamkeit für den Islam als politischen Faktor ist auch das Interesse an den 'missionarischen' Erfolgen des Islams im Westen wach geworden (vgl. Poston 1992). Eine Menge autobiographischer oder programmatischer Konversionsliteratur ist in den letzten Jahren erschienen oder neu aufgelegt worden (Hofmann 21993; Asad 1992; Garaudy 1992 u.v.a.), Talkshows luden Konvertiten zum Gespräch, und Tageszeitungen und Journale lancierten hohe Zahlen und starke Zuwachsraten. Man bekommt aufgrund dieser Medienresonanz den Eindruck, als ob der Konversion zum Islam im Bereich der Lebensführung vergleichbare Relevanz zugemessen würde wie im Bereich der Politik dem islamischen Fundamentalismus.

Dabei gilt die öffentliche Aufmerksamkeit vor allem den konvertierten Frauen, zumal dort, wo sie sich Elemente der islamischen Kleidung, wie Kopftuch oder Schleier, zu eigen machen. Verweist doch das äußere Erscheinungsbild vieler Konvertitinnen unübersehbar darauf, daß sich mit der Übernahme der Religion auch eine bestimmte

\footnotetext{
${ }^{1}$ Dieser Aufsatz basiert auf einem Vortrag, der im Oktober 1994 im Rahmen der Sektion Kultursoziologie auf dem Kongreß der DGS in Halle gehalten wurde. Für kritische Hinweise bei der Überarbeitung danke ich dem Herausgebergremium der ZfS, Hilge Landweer und Werner Sahr, sowie den anonymen Gutachtern zu meinem DFGAntrag, deren Anmerkungen auch für diesen Text hilfreich waren.
}

Form der Lebensführung verbindet, bei der die Symbolisierung des Geschlechterverhältnisses eine zentrale Rolle spielt.

So vielschichtig die Bedeutung weiblicher Kopfbedeckung im Orient sein mag (s. Colpe 1989), im europäischen Kontext von konvertierten Europäerinnen getragen, bekommt sie den Charakter eines Musterbeispiels (Kuhn 1988: 392) für die Aneignung einer fremden Religion und Kultur ${ }^{2}$ mit den dafür charakteristischen Grenzziehungen zwischen den Geschlechtern und der gleichzeitig stärkeren Durchdringung von Religion und Alltagsleben. Dies wirkt in sich als säkular verstehenden und programmatisch auf Gleichheit zielenden Kulturen offenkundig als Provokation, weshalb nicht zufällig das Kopftuch immer wieder zum Gegenstand heftiger Auseinandersetzungen wird (vgl. Thömmes 1993). Jedenfalls hat es bei westlichen Konvertitinnen nichts von einer traditionalen Selbstverständlichkeit, sondern den Charakter einer gezielt eingesetzten präsentativen Symbolik, ${ }^{3}$

${ }^{2}$ Es geht dabei in erster Linie um einen Proze $B$ von Identifikation und Abgrenzung, in dem „Eigenes“ und „Fremdes" voneinander unterschieden wird. Insofern sind Hinweise darauf, daß das Kopftuch ursprünglich aus dem byzantinischen Christentum in den Islam importiert worden sei, oder auf die Verwendung des Kopftuchs in nordeuropäischen Trachten zwar informativ, treffen aber letztlich nicht die Logik des Vorgangs dieser Identifikation.

${ }^{3}$ Der Begriff der prasentativen Symbolik wird hier im Anschluß an Susanne Langer (1984) verwendet.

Die neuere Literatur zeigt, daß der reflexive Gebrauch traditioneller Symbole auch in originär islamischen Kontexten zunimmt. So betont Helen Watson (1994), daß das 
wie sie in dieser Form im Kontext der christlichen Kirchen in Westeuropa kaum noch anzutreffen ist.

Bei männlichen Konvertiten ist ein solcher $\mathrm{Zu}$ sammenhang weniger augenscheinlich. Dennoch übernehmen auch sie mit dem Islam häufig nicht allein den Glaubenskodex, sondern ebenfalls die kulturelle Codierung des Geschlechterverhältnisses. Entsprechend ist - will man sich nicht mit dem schnell verfügbaren Etikett des „Patriarchalen“ begnügen - auch hier nach der Sinnstruktur dieser Aneignung zu fragen. Auch männlichen Konvertiten steht für diese Seite des Aneignungsprozesses im übrigen eine präsentative Symbolik zur Verfügung: die Beschneidung.

Die folgenden Überlegungen setzen an diesem Zusammenhang von Religion und Lebensführung an und entwickeln davon ausgehend eine Erklärung für eine Variante der Konversion deutscher Frauen und Männer zum Islam. Es geht also nicht um einen generellen Erklärungsanspruch: Was hier im Rückgriff auf mehrere Fälle der Konversion zum Islam herausgearbeitet werden soll, ist ein bestimmter Typus der Konversion, bei dem die mit dem Islam verbundene Symbolisierung von Geschlechtsrolle und Geschlechterverhältnis sinnstrukturell verknüpft ist mit der Bewältigung einer biographischen Problemlage. Diese besteht - das soll im folgenden gezeigt werden - in der Erfahrung von Normverletzungen auf dem Gebiet der Sexualität und der damit einhergehenden sozialen Entwertung. Der Konversion zum Islam - so die These - kommt in diesen Fällen die Funktion zu, das Problem von Normverletzung und sozialer Entwertung auf eine spezifische Weise zu bewältigen. Dies geschieht auf dem Wege symbolischer Transformation (s. Langer 1987), durch die biographische Erfahrungen einerseits ,präsentiert" ${ }^{* 4}$ werden, gleichzeitig aber durch neue Rahmungen oder durch Verschiebungen in den religiösen Sinnbereich ihre konkrete, personal verankerte lebensgeschichtliche Problematik verlieren.

Über den hier behandelten Typus hinaus sind Prozesse symbolischer Transformation problematischer biographischer Erfahrung generell charakteristisch für die Konversionsphänomene, die im Rahmen meiner Untersuchung vorzufinden sind.

\footnotetext{
Tragen des hijab auch für originär moslemische Frauen den Charakter des Selbstverständlichen, aber auch Beliebigen zunehmend verliert und zum bewußt gewählten Symbol wird.

4 „Präsentiert" bezieht sich hier sowohl auf den zeitlichen Aspekt - im Sinne von Vergegenwärtigung - als auch auf den Aspekt der symbolischen Darstellung.
}

Die Probleme, um die es dabei geht und die Lösungen, die sich über den Islam vermitteln, sind jedoch verschieden. Bei einem anderen Typus etwa ist das biographische Problem durch eine (negative) Verlaufskurvendynamik im Kontext politischer Gesinnungsgemeinschaften (und deren kultureller Ausläufer) bestimmt, die - vorzugsweise im Sufismus - als ,Weg“ und Teil einer „Führung“ präsentiert werden. Dabei ist es die Bindung an einen religiösen Führer - einen ,Sheik“ -, durch die alte Bezüge abgestreift werden, die Struktur hochgradiger innerer Verpflichtung mit der Folge einer tendenziellen Abkehr von alltagspraktischen Verbindlichkeiten aber religiös codiert und damit auf die Spitze getrieben wird.

\section{Perspektiven einer Soziologie der Konversion}

\subsection{Zur Soziologie radikalen Wandels}

Der Kern aller Konzeptionen von Konversion - so resümieren David Snow und Michael Machalek (1983: 261) - sei die Idee radikalen Wandels. Wenn auch einzelne Untersuchungen in diesem Bereich bei der Analyse dieses Wandels unterschiedliche Akzente setzen, so besteht doch breite Übereinstimmung darüber, daß es sich bei Konversionen um einen umfassenden Wandel der Identität handelt, im Zuge dessen die bisherige realitätsstrukturierende Weltsicht zugunsten einer anderen aufgegeben wird (Lofland/Stark 1965; Jones 1977; 1978; Snow/Machalek 1983; 1984; Sprondel 1985; Ingram 1989; u.a.), mit nachhaltigen Folgen für das Verhalten der jeweiligen Person, ihren sozialen Kontext etc. ${ }^{5}$

Aufgrund dieser Konzeptualisierung von Konversion stand zumindest die soziologische Konversionsforschung schon seit den 60er Jahren in einer gewissen Wahlverwandtschaft $\mathrm{zu}$ einem allgemeinen Religionsbegriff, da so verstandene Konversionsphänomene nicht auf Religion im engeren Sinne zu beschränken waren, sondern sich auf alle Ideologien bezogen, denen die Leistung der „Kosmisierung" (Berger 1988: 25) zukam (s. Sprondel 1985: 557).

\footnotetext{
${ }^{5}$ Snow und Machalek (1984: $173 \mathrm{f}$.) identifizieren diesen Wechsel anhand folgender Merkmale: der Rekonstruktion der Biographie nach Maßgabe des neuen ,universe of discourse"; der Übernahme eines neuen, leitenden Attributionsschemas; der Suspension analoger Begründungen; sowie der Annahme der Konvertitenrolle in allen interaktiven Situationen.
} 
Die Theoriebezüge, auf die bei der Analyse solcher Wandlungsprozesse rekurriert wurde, zeichneten sich ursprünglich dadurch aus, daß sie den Zusammenhang von „Weltsicht" und Praxis- bzw. Erfahrungsform betonten. Dies gilt für die Konzeptualisierung im Anschluß an Mead, Konversionen als Wechsel des „universe of discourse“ zu betrachten, der ja dort als „gemeinsamer gesellschaftlicher Erfahrungs- und Verhaltensprozeß" (Mead 1973: 129) definiert ist; wie auch beim Rekurs auf das Kuhn'sche Konzept des Paradigmawechsels, bei dem ebenfalls die mit einer veränderten disziplinären Matrix verbundenen konkreten Formen der Problemlösung (Kuhn 1978: 393) mitgedacht sind.

Dieser Zusammenhang von Weltsicht und Praxisbzw. Erfahrungsform - oder auch: von Ideologie und Lebensführung - rückte im Zuge der arbeitsteiligen Bearbeitung von Konversionsphänomenen wieder in den Hintergrund (vgl. dazu auch Richardson 1978) oder wurde lediglich programmatisch vorausgesetzt, aber nicht mehr eigens analysiert. Die Forschung konzentrierte sich zunehmend auf Konversionsursachen und -abläufe einerseits und auf die Formen der Darstellung religiösen Wandels andererseits.

Verstärkt wurde diese Trennung von 'Wissensanalyse' und 'Verlaufsanalyse' durch eine Entwicklung, die Krech (1995: 146) als „,linguistic turn“ der soziologischen Konversionsforschung bezeichnet hat: durch die zunehmende Konzentration auf das kommunikative Geschehen der Konversion, die einherging mit der methodologisch begründeten Frage nach dem Status der Konversionserzählungen als Datenquellen. In dem Maße, wie die Aufmerksamkeit dafür wuchs, daß es sich bei Konversionserzählungen um eine spezifische kommunikative Gattung (Ulmer 1988; 1990; s. auch Luckmann 1986) handelt, die möglicherweise weniger als Bericht über Veränderung (,account-giving“), sondern eher als Behauptung einer Veränderung (,claims-making“) anzusehen ist (Kilbourne/Richardson 1988), schien eine Rekonstruktion von Ereignisfolgen und Positionssequenzen (,Konversionskarrieren“) aufgrund dieser Quellen zunehmend problematisch oder auch von geringem soziologischen Interesse. Die Fokussierung der Aufmerksamkeit für die Wissens- oder Kommunikationsform ließ die Praxis- und Erfahrungsform, auf die dabei referiert wurde, in den Hintergrund treten.

${ }^{6}$ Kuhn (1978) unterscheidet diese beiden Sachverhalte später begrifflich als ,paradigm“ und „exemplar“.

\subsection{Erweiterung der Perspektiven eines funktionalen Zugangs}

Mit der wissenssoziologischen Analyse von Konversionen verbindet sich eine funktionale Perspektive, insofern etwa kommunikative Gattungen als Lösung bestimmter kommunikativer Probleme angesehen werden. Ich werde an diese funktionale Perspektive hier anschließen, sie allerdings auf den Kontext der Biographie beziehen.

Eine funktionale Perspektive aus wissenssoziologischer Sicht ist unter anderem von Walter Sprondel (1985) vertreten worden, der Konversionen als radikalen Wandel der Struktur subjektiver Weltsichten definiert. Dabei kommt der Konversionserzählung in doppelter Hinsicht die Funktion der Lösung kommunikativer Probleme zu: Zum einen geht es um das Problem von Individuen, inneres Erleben - eine außeralltäglich erzeugte Erschütterung und den daraus resultierenden inneren Wandel - nach außen hin glaubhaft zu machen. Zum anderen geht es um das Problem von Weltanschauungsgruppen, die von ihnen lediglich verheiBene "Neuordnung der Welt" durch den individuellen Wandel ihrer Anhänger bereits in der Gegenwart überzeugend zu repräsentieren. Den Konversionserzählungen kommt dabei die Funktion zu, individuelles Erleben zu objektivieren und mit der glaubwürdigen Darstellung des inneren Wandlungsprozesses gleichzeitig die Glaubwürdigkeit der Weltanschauungsgruppe verläßlich zum Ausdruck zu bringen.

In dieser Perspektive geht es also um die Frage nach der kommunikativen Funktion von Konversionserzählungen. Was der wissenssoziologische Zugang jedoch nicht beantwortet, ist die Frage, wie es überhaupt zu solchen kommunikativen Problemen kommt, was also Personen veranlaßt, ihre bisherige Weltsicht in einer so radikalen Weise zu verändern, daß eine umfassende Veränderung ihrer sozialen Umwelt, ja der zentralen Dimensionen ihrer Identität daraus resultiert.

In einer auf die Biographie erweiterten funktionalen Perspektive formuliert: Welche Funktion erfillt die Konversion in der Biographie oder: welches Problem der Biographie wird durch den radikalen Wandel der Weltsicht gelöst?

Die Anwendung eines funktionalen Zugangs beim Versuch einer biographischen Erklärung von Konversionen macht drei Hinweise erforderlich:

1. Wenn hier von einer „Funktion für die Biographie" die Rede ist, sind damit nicht subjektive von den Konvertiten selbst als Erklärung vorge- 
brachte - Motive gemeint, sondern das, was im Rahmen der funktionalen Analyse Mertons als „latente Funktionen“ (Merton 1995: 49), bzw. im Kontext der Methodologie der „Objektiven Hermeneutik", auf die hier Bezug genommen werden soll, als "latenter Sinn“ (Oevermann u.a. 1979: 367) bezeichnet wird.?

2. Ein so definierter funktionaler Zugang impliziert keine Vorstellung von kausaler Notwendigkeit, sondern die Annahme, daß es für die betreffende Selektion - also in diesem Fall für die vollzogene Konversion - auch funktionale Äquivalente gibt (vgl. Merton 1985: 50; Luhmann 1970: 33). Die Konversion zur Religion eines bestimmten Typs wird demnach nicht als Resultat einer Zwangsläufigkeit betrachtet, die von vornherein nur die Wahl dieser einen Alternative möglich gemacht hätte. Die Betonung von Zwangsläufigkeit kann allerdings ein Element in der Selbstdarstellung von Konvertiten sein. Und sie kann - das zeigt der biographische Zugang - durch die Engführungen und daraus resultierenden Bindungswirkungen entstehen, die biographische Selektionen mit sich bringen. Wenn also eine Person zum Islam konvertiert, so heißt das nicht, daß nicht andere Weltanschauungsgruppen für sie dieselbe Funktion hätten erfüllen können. $\mathrm{Zu}$ zeigen ist allerdings, wie es $\mathrm{zu}$ dieser spezifischen Selektion kommt und welche Plausibilität sie für sich in Anspruch nehmen kann.

3. Bei Biographien den Ausgangspunkt zu nehmen, bedeutet nicht die Beschränkung auf Singuläres und den Verzicht auf Generalisierung. Es geht vielmehr um den Versuch, eine funktionale Erklärung 'von unten nach oben' aufzubauen: d.h. ausgehend von der Analyse der Sinnstruktur einzelner Fälle die Frage nach der typischen Struktur dieser Lösungen und weitergehend die Frage nach den allgemeineren Funktionen der Konversion zur Religion einer anderen Kultur zu stellen. ${ }^{8}$

\footnotetext{
${ }^{7}$ Es gibt eine Reihe von Entsprechungen zwischen funktionaler Analyse und objektiver Hermeneutik, die eine solche Verbindung sinnvoll erscheinen lassen: 1. Das, was im Rahmen der funktionalen Analyse als ,funktionales Äquivalent" bezeichnet wird, findet in der objektiven Hermeneutik eine Entsprechung in der gedankenexperimentellen Konstruktion „objektiver Möglichkeiten“, mit denen eine konkrete Problemstellung gelöst werden kann. Der ,latenten Funktion“ im Konzept Mertons, mit der entsprechenden Abgrenzung gegenüber dem ,subjektiven Motiv" entspricht bei Oevermann die Unterscheidung von „objektiver Bedeutung“ bzw. „latentem Sinn“ und „subjektiv gemeintem Sinn".

${ }^{8}$ Vgl. hierzu etwa die Bemerkungen Robert Mertons (1995: 40-42) zur Funktion von Minderheitenreligionen.
}

Dies zielt auf den Zusammenhang von biographischer Identität und kollektiven Symbolsystemen. $\mathrm{Zu}$ beantworten wäre am Ende, welche Elemente dieser Religion sich mit welchen typischen biographischen Problemlagen vermitteln und warum westlich sozialisierte Personen Problemlösungen gerade in einer 'fremden' Religion und Kultur finden.

\subsection{Methodische Folgerungen}

Der skizzierte Zugang hat methodische Konsequenzen: Die Frage nach latenten Funktionen bzw. latentem Sinn setzt ein Verfahren voraus, das mehr zu erheben in der Lage ist als subjektive Theorien und die Argumentationsmuster von Weltanschauungsgruppen. Bereits für die Erhebung folgt daraus, nicht auf Konversionserzählungen im engeren Sinne abzustellen, sondern breitere biographische Erzählungen zu generieren, um überhaupt die Dynamik von Stegreiferzählungen in Gang zu setzen, wie sie Fritz Schütze (1976) herausgearbeitet hat. Es geht also darum, eine Form der Darstellung zustande zu bringen, die die Interviewpartner veranlaßt, Situationen und Konfliktlagen im Umfeld der Konversion auf eine Weise zu rekapitulieren, die über das ,Skript Konversionserzählung " hinausgeht. ${ }^{9}$

Ich will die Differenz zwischen Konversionsgeschichte und biographischer Erzählung an einem Beispiel verdeutlichen:

Einer meiner Probanden, dem in der islamischen Gemeinschaft seiner Stadt gewisse Prominenz zukam, leitete das Interview mit dem Hinweis ein, $\mathrm{da} ß$ er solche Gespräche häufiger führe und im übrigen seine Konversionsgeschichte bereits schriftlich verfaßt habe, so daß wir uns im Interview an diesem Manuskript orientieren könnten.

Hier wird etwas verbalisiert, was für andere Interviews teilweise, wenn auch weniger deutlich, auch gilt: daß es verfestigte subjektive Theorien und fixierte Geschichten zum Thema der eigenen Konversion gibt, in denen gewisse Details beleuchtet werden, andere dagegen völlig ausgespart bleiben. Der genannte Fall ist methodisch insofern interessant, als hier eine schriftlich verfaßte Konversionsgeschichte und ein biographisches Interview vor-

\footnotetext{
9 Die bisherigen Erfahrungen aus Interviews mit Konvertiten zeigen, daß dies in der Tat möglich ist, allerdings auch ein differenziertes Interviewerverhalten erfordert: Stegreiferzählungen müssen bei verschiedenen Probanden auf sehr verschiedene Weise zutage gefördert werden.
} 
liegen und insofern die spezifische Selektivität ${ }^{10}$ der Konversionsgeschichte erkennbar wird.

Im vorliegenden Fall gibt es in der Konversionsgeschichte eine deutliche Zäsur zwischen einem ersten überwiegend biographischen Teil und einem zweiten Teil, der von der Biographie weitgehend abstrahiert und wesentlich aus religiösen Argumentationen mit einer Fülle von Koranzitaten besteht. Die Zäsur zwischen beiden Teilen - gewissermaßen den Übergang zwischen vorkonversioneller Biographie und nachkonversioneller, 'biographieloser' religiöser Existenz - markiert folgender Satz: „Nachdem ich meine Berufsausbildung abgeschlossen hatte, veranlaßte mich meine immer stärker werdende Sehnsucht nach moralischem Handeln und nach dem Sinn des Lebens mich mit der Frage nach der Existenz Gottes auseinanderzusetzen." 11

In der schriftlichen Konversionsgeschichte bleibt völlig offen, wie es zu dieser ,immer stärker werdenden Sehnsucht nach moralischem Handeln" kommt. Das biographische Interview gibt hierüber mehr Aufschluß: Der Proband schildert ein Leben mit exzessivem Konsum von Alkohol und Sexualität. Gewissermaßen 'verpackt' in abstrakte moralische Erwägungen, aber dennoch mit unverkennbarem lebensgeschichtlichen Bezug, nimmt das Problem des Ehebruchs im Interview breiten Raum ein. Deutlich wird dabei die Dramatik, in die die Biographie einmündet: Während der Proband zum Islam konvertiert und darüber sein Leben neu strukturiert, dramatisiert sich über viele Jahre hinweg der Alkoholismus seiner Ehefrau, bis sie schließlich daran stirbt.

Ohne diesen Fall hier im Einzelnen interpretieren zu wollen, wird doch daran der unterschiedliche Charakter von Konversionsgeschichte und biographischer Erzählung deutlich. Die Konversionsgeschichte liefert mit dem Stichwort: 'Sehnsucht nach einem moralischen Leben' lediglich einen groben Anhaltspunkt für den biographischen Umbruch und verweist in ihrer sprachlichen Struktur gleichzeitig darauf, daß die Konversion gewissermaßen ein Umschalten vom 'Modus Biographie' auf den 'Modus Religion' impliziert. Sie blendet aber die zentrale Dynamik aus, aus der heraus sich

\footnotetext{
${ }^{10}$ Natürlich handelt es sich beim biographischen Interview ebenfalls um eine selektive Vergegenwärtigung der Lebensgeschichte, allerdings um eine anderen Charakters.

11 Insofern findet sich auch hier die charakteristische Dreiteilung von Konversionsgeschichten, wie sie von Ulmer (1988) als Gattungsmerkmal herausgearbeitet wurde.
}

dieses Umschalten entwickelt und substituiert diese durch das abstrakte Motiv der Moralsuche und den Hinweis auf religiöse „Zeichen“. Im Text heißt es hier: ,Ich betete um ein Zeichen von ihm. Wenn es dich gibt oh Gott, so betete ich, dann laß mich am kommenden Wochenende eine unbedeutende Summe im Lotto gewinnen. Zu meiner Überraschung gewann ich einen Betrag von über drei DM. Das war für mich ein Anfang an Gott zu glauben und ich begann ihn zu suchen."

\section{Zur empirischen Erhebung}

Die Überlegungen dieses Artikels stützen sich auf eine laufende empirische Untersuchung über Konversion zum Islam in der Bundesrepublik, die gegenwärtig gerade durch eine vergleichend angelegte Untersuchung in den Vereinigten Staaten ergänzt wird. ${ }^{12}$

\subsection{Die Strukturierung des Phänomens}

Konversionen von Deutschen zum Islam sind - in auffälligem Gegensatz zur Medienresonanz - im Vergleich zu den in der Bundesrepublik lebenden Migranten islamischen Glaubens ein marginales Phänomen. Die Daten der Volkszählung von 1987 weisen für die alte Bundesrepublik eine Zahl von 1.650.952 Muslimen aus. Darunter befinden sich 47.966 Deutsche. ${ }^{13}$ Allerdings sind deutsche Muslime nicht, wie immer wieder fälschlich unterstellt wurde, identisch mit Konvertiten. Die Zahlen, die in diversen Journalen ${ }^{14}$ die Runde machten, beruhten offensichtlich auf einer solchen irrtümlichen Gleichsetzung (vgl. auch Leggewie 1993: 126).

Es ist ausgesprochen schwierig, das Phänomen von Konversionen zum Islam in Umfang, Struktur und

12 Die Erhebung in den USA wird von der University of Berkeley, California, aus organisiert. Eine Frage dabei wird sein, ob bei Konversionen zum Islam in den Vereinigten Staaten, die auffälligerweise vor allem unter der afroamerikanischen Bevölkerung verbreitet sind, ebenfalls Erfahrungen sozialer Entwertung eine Rolle spielen und wenn ja - in welcher Weise diese Erfahrungen religiös transformiert werden.

${ }^{13}$ Nach Angaben des Islam-Archivs Deutschland liegt die aktuelle Zahl bei ca. 62.000 .

14 Laut SPIEGEL (8/1993: 74) sind "mehr als 50000 deutsche Frauen zum Islam konvertiert". In einer anderen Ausgabe (14/1992: 38) ist von 100000 deutschen Muslimen die Rede. Die Frauenzeitschrift marie claire (Schwabenthan 1990) sprach zum gleichen Zeitpunkt von 50000 deutschen Konvertiten. Die Quellen bleiben dunkel. 
Entwicklung korrekt einzuschätzen. Aufgrund der nicht-kirchlichen Verfaßtheit des Islams und der Einfachheit der Konversion ${ }^{15}$ werden Konversionen zum Islam häufig nirgendwo aktenkundig.

Gewisse Anhaltspunkte über die Zusammensetzung der Konvertiten erhält man durch das IslamArchiv in Soest, ${ }^{16}$ einer Stelle, die Dokumente über Konversionen und islamische Heiraten ausstellt.

Nach Angaben des Archiv-Leiters liegt das durchschnittliche Konversionsalter bei 27 Jahren, was in der Tendenz mit einer amerikanischen Untersuchung (Poston 1992) ${ }^{17}$ und eigenen Auswertungen von Konversionsakten der Berliner „Gemeinschaft deutscher Muslime“18 übereinstimmt, die beide zu dem Ergebnis kommen, daß Konversion zum Islam überwiegend kein Adoleszenz-Phänomen ist. Begreift man Konversionen funktional als „Problemlösungen“, so folgt daraus, daß es in den meisten Fällen nicht die charakteristischen Adoleszenzkrisenphänomene sein werden, die bei der Entscheidung zur Konversion eine Rolle spielen, sondern Probleme von Erwachsenen, die bereits einige Erfahrungen in den Bereichen von beruflicher Bildung und Arbeit, von Partnerschaft und Familie gesammelt haben.

Die Auswertung der Konversions-Akten der Berliner „Gemeinschaft deutscher Muslime“ ergab für den Zeitraum von 1988 bis September 1993 eine Anzahl von 118 an dieser einen Stelle registrierten Konversionen, ${ }^{19}$ davon 50 Männer und 68 Frauen.

Etwa jede dritte Konversion eines Mannes und knapp jede zweite Konversion einer Frau stand in einem direkten zeitlichen Zusammenhang mit einer islamischen Heirat. Allerdings ist davon auszu-

15 In der Regel wird lediglich vor zwei Zeugen die Shahada, das Bekenntnis zu Allah als dem einzigen Gott gesprochen.

${ }^{16}$ Allerdings sind auch diese Angaben nicht wirklich wissenschaftlich kontrolliert, da das Islam-Archiv entgegen dem eigenen Anspruch offenbar eher den Charakter eines Privatarchivs hat und für Wissenschaftler nicht zugänglich ist.

${ }^{17}$ Diese Untersuchung erfaßt allerdings - entgegen dem, was sie suggeriert - nicht Verteilungen unter muslimischen Konvertiten in den USA, sondern wertet lediglich Konversionsberichte in muslimischen Zeitschriften im Hinblick auf die dort gemachten Angaben über Geschlecht, Alter bei der Konversion etc. aus.

${ }^{18}$ Diese Auszählung ergab bei den Frauen ein durchschnittliches Konversionsalter von 30, bei den Männern von ca. 36 Jahren.

19 In 38 Fällen handelte es sich um nachträgliche Registrierung bereits länger vollzogener Konversionen. gehen, daß es darüber hinaus auch in vielen anderen Fällen einen Zusammenhang zwischen Konversion und der Verbindung mit einem Partner gibt, der dem islamischen Glauben angehört.

Dies sagt jedoch zunächst nur etwas über die Kontextuierung, nicht unbedingt etwas über den Sinn der Konversion aus. Im Zusammenhang mit Eheschließungen vorgenommene Konversionen können rein instrumentelle Anpassungsvorgänge sein, es kann aber auch umgekehrt gerade die Ehe der Ort sein, an dem verbindlich - und sozial sanktioniert - eine umfassende religiöse und kulturelle Neuorientierung vonstatten geht. Für eine solche sinnstrukturelle Verschränkung von Konversion und Eheschließung spricht auch die wissenssoziologische Erkenntnis, daß sich im Zuge verbindlicher Paarbildungen ohnehin eine Art „Konversion" hin zu einer gemeinsamen neuen Weltsicht vollzieht (Berger/Kellner 1965). Vielleicht sind Veränderungen der ,subjektiven Weltsicht" (Sprondel 1985) dort am nachhaltigsten, wo sie auch im intimen Kontext repräsentiert, bestätigt und sanktioniert werden.

Befunde qualitativer Studien aus der amerikanischen Islam-Forschung unterstützen die Annahme einer sinnstrukturellen Verschränkung von Konversion und Eheschließung. Yvonne Haddad (1986: 7) etwa weist darauf hin, daß Konversionen zum Islam häufig bereits stattfinden, bevor die Konvertierten einen Partner finden, der dieser $\mathrm{Re}$ ligion angehört, d.h. daß beide Phänomene - Konversion und Partnerwahl - Ausdruck desselben Prozesses biographischer Neuorientierung sind. Den Sinn solcher Prozesse gilt es hier zu rekonstruieren.

\subsection{Das Sample}

Die empirische Erhebung wurde in mehreren westdeutschen Städten durchgeführt. Sie umfaßt bisher neunzehn Probanden - elf Frauen und acht Männer - mit denen jeweils mehrstündige biographische Interviews durchgeführt wurden. Fünf Probanden - zwei Frauen und drei Männer - gehören dem Sufismus an, vierzehn Befragte zählen sich zu verschiedenen Schulen des sunnitischen Islam. Die Dauer der Zugehörigkeit zum Islam variiert zwischen einigen Monaten und über dreißig Jahren, meist waren die Probanden jedoch bereits seit mehreren Jahren Muslime. Eine Probandin hatte sich zum Zeitpunkt des Interviews vom Islam wieder abgekehrt und war dabei, sich von ihrem muslimischen Ehemann zu trennen. Der sozi- 
ale Status der Interviewees variiert vom ungelernten Arbeiter über die Kindergärtnerin bis hin zum Universitätsprofessor. In zwei Fällen liegt neben dem biographischen Interview auch eine schriftliche Konversionsgeschichte vor, die die Befragten in einem anderen Kontext zu Zwecken der AuBendarstellung, aber auch der Verständigung innerhalb der islamischen Gemeinschaft verfaßt hatten.

Bei der Zusammensetzung des Samples kamen verschiedene Kriterien zum Tragen: zunächst ging es darum, aufgrund äußerer Merkmale eine möglichst vielfältige Zusammensetzung der Untersuchungsgruppe zu ermöglichen - im Hinblick auf Alter, Geschlecht, sozialen Status, Dauer der Religionszugehörigkeit und der Verortung innerhalb des islamischen Spektrums. Dabei konnte es nicht darum gehen, die - ohnehin nicht bekannte - statistische Verteilung in der Grundgesamtheit durch Fälle abzubilden, sondern lediglich darum, die Wahrscheinlichkeit dafür zu erhöhen, daß durch die einbezogenen Fälle unterschiedliche Sinnstrukturen repräsentiert sein würden.

Der Zugang zum Feld wurde sowohl über einzelne Personen als auch über bestimmte Institutionen gesucht: auf manche Personen wurde ich durch berufliche Kontakte hingewiesen; andere sprach ich bei Treffen von deutschen Muslimen an, wie es sie in mehreren Städten gibt; wieder andere bei verschiedenen Veranstaltungen über den Islam. Ausgehend von diesen Kontakten wurden dann im Schneeballsystem weitere Personen ermittelt, wobei zunehmend Kriterien eines ,theoretical sampling" (Glaser/Strauss 1967) zum Tragen kamen. Ein solches Kriterium, das sich im Lauf der ersten Interviews mit weiblichen Konvertiten als äußeres Merkmal von hoher symbolischer Bedeutung herausstellte, war etwa, ob die Probandinnen islamische Kleiderregeln, vor allem das Tragen des Kopftuchs, akzeptierten oder nicht. Ein anderes Selektionskriterium war, ob die Konvertiten den Islam nach außen hin institutionell repräsentierten oder ob ihre religiöse Haltung eher ,privaten“ Charakter zu haben schien. Die Grenzen eines solchen ,theoretical sampling" bestehen allerdings darin, daß äußere Merkmale allenfalls grobe Indikatoren für biographische Sinnstrukturen sind und sich als Unterscheidungsmerkmale im Nachhinein manchmal als untauglich herausstellten. Aufgrund persönlicher Hinweise wurden dann noch gezielt einzelne Personen in das Sample mit einbezogen, die einen maximalen Kontrast oder auch große Nähe zu den bisherigen Fällen zu repräsentieren schienen.
Die Interviews werden nach dem Verfahren der objektiven Hermeneutik ausgewertet, was sequenzanalytische Interpretationen der biographischen Daten sowie von Teilen des Interviewmaterials einschließt.

Die Analyse zielt auf eine Typenbildung, bei der die biographische Funktion der Konversion die zentrale Dimension der Typisierung darstellt.

\section{Konversion als symbolische Transformation problematischer Erfahrung}

Im folgenden soll es darum gehen, anhand von drei Fällen einen Typus der Konversion zum Islam herauszuarbeiten, bei dem - so die These - der Konversion die Funktion zukommt, biographisch einschneidende Erfahrungen der Verletzung von Sexualnormen und der damit verbundenen sozialen Entwertung zu bewältigen. ${ }^{20}$

Wie bereits ausgeführt wurde, entspricht es einem funktionalen Zugang davon auszugehen, daß die Lösung der genannten Probleme auch über funktionale Äquivalente zur Konversion möglich (gewesen) wäre. Insofern enthalten Konversionen zum Islam meist ein Moment von Kontingenz, häufig repräsentiert durch die Begegnung mit einer Person, die den Islam als Möglichkeit überhaupt in den eigenen Horizont einführt. Hätte es diese Begegnungen nicht gegeben, wären die hier zur Debatte stehenden Biographien vermutlich anders verlaufen.

Dennoch stellt der Islam ${ }^{21}$ ein spezifisches Instrumentarium bereit, das die Konversion als Lösung für die genannten Probleme in besonderer Weise geeignet macht:

1. Er erscheint westlichen Beobachtern in einer im Vergleich zu den im eigenen Kontext dominanten Religionen - engen Verschmelzung von Religion und Lebensführung, gerade im Hinblick auf die Regelung des Geschlechterverhältnisses.

2. Er hält mit Kopftuch und Beschneidung religiös zwar nicht unbedingt verpflichtende, aber kulturell mit dem Islam häufig eng verbundene

20 Zum Zusammenhang von Symbol und Erfahrung im Kontext von Konversionen s. auch die Arbeiten von Peter Stromberg (1991).

${ }^{21}$ Hier geht es nicht um die Festschreibung eines einheitlichen Islam (vgl. dazu Al-Azmeh 1993), sondern um das Spektrum der Möglichkeiten, die darin bereitliegen und von Konvertiten selektiv angeeignet werden. 
präsentative Symbole bereit, die in ihrem symbolischen Gehalt auf die Codierung des Geschlechterverhältnisses zielen.

3. Er ermöglicht gerade durch diese enge Verbindung 'religiöser' und 'geschlechts-kultureller' Momente die symbolische Transformation von Erfahrungen im Bereich der Sexualität in den Bereich der Religion hinein. Insofern präsentiert die "äußere Erscheinungsform" der Konvertiten in symbolischer Weise auch die Probleme, die mit der Konversion gelöst werden.

\subsection{Fall I: Von sexueller Normverletzung zu religiös begründeter Moral}

\subsubsection{Biographische Skizze}

Habib (Paul) Bode stammt aus einem typischen Mittelschichts-Milieu - sein Vater war Angestellter, die Mutter lange Zeit Hausfrau. Die Eltern kannten sich seit ihrer Jugend, bekamen aber erst spät ihr einziges Kind. Als Paul elf Jahre alt war, Anfang der 70er Jahre, begann seine Mutter eine Affäre mit einem Tunesier. Während sie selbst eine Trennung von ihrem Ehemann eigentlich nicht intendierte, drängte Pauls Vater auf eine Klärung der Verhältnisse und zog schließlich aus der Wohnung aus. An seine Stelle trat der Liebhaber seiner Frau. Während Pauls Eltern sich scheiden ließen, zog der Tunesier, der in seinem Heimatland Frau und Kinder hatte, eine Scheidung nicht in Erwägung. Er war gebürtiger Muslim, ohne jedoch diesen Glauben und die damit verbundenen Regeln zu praktizieren.

Als Paul dreizehn war, bekam die Mutter von ihrem Freund einen weiteren Sohn. Dieser wurde von seinem Vater adoptiert, erhielt einen arabischen Namen und wurde nach muslimischer Sitte als Kind beschnitten.

Etwa zu dieser Zeit fing Paul an, sich intensiv mit dem Herkunftsland des Stiefvaters und der Religion des Islams auseinanderzusetzen. Er gab sich gegenüber den Verwandten des Stiefvaters als Muslim aus und ließ sich von diesem einen arabischen Namen geben. In der Schule bestand er darauf, als „Habib“ angesprochen zu werden. Mit vierzehn weigerte er sich, sich konfirmieren zu lassen und trat mit fünfzehn offiziell zum Islam über. Noch während seiner Schulzeit ließ er sich beschneiden und änderte als Erwachsener auch offiziell seinen Vornamen. In der Zwischenzeit hatte seine Mutter sich von ihrem Lebensgefährten we- gen seiner Frauenaffären wieder getrennt. Ihren jüngeren Sohn ließ sie später taufen und konfirmieren.

Ein gläubiger Bruder seines Stiefvaters übernahm bei Habib die Rolle eines religiösen Lehrers. Habib reiste mehrfach nach Tunesien zur Familie seines Stiefvaters. Er wollte sich dort sogar nach der Sitte des Landes mit einer jungen, ihm nur vom Ansehen bekannten Frau verheiraten lassen, nahm jedoch wegen des hohen Brautpreises davon wieder Abstand. Schließlich heiratete er in Deutschland eine Kurdin, mit der er mittlerweile fünf Söhne hat.

Sein Halbbruder ist im Unterschied zu ihm nur an „deutschen, blonden Mädels" interessiert, hat keinerlei Interesse am Islam, benutzt bisweilen einen falschen deutschen Vornamen und will versuchen, seinen islamischen Namen wieder abzulegen.

\subsubsection{Verletzung sexueller Normen vs. Verstoß gegen religiöse Konvention}

Die biographischen Daten, die hier versammelt sind, kommen erst im Verlauf des gesamten Interviews aufgrund von Nachfragen zustande, die den Interviewee zu Detaillierungen und Plausibilisierungen veranlassen. Dagegen zeichnet sich der erste Durchgang der biographischen Erzählung dadurch aus, daß die brisanten biographischen Ereignisse und die daraus resultierenden Konsequenzen mit Ausnahme weniger unverzichtbarer Eckpunkte ausgeblendet bleiben. Die Scheidung der Eltern wird zwar erwähnt, bleibt aber völlig unmotiviert, der tunesische Freund der Mutter tritt vermeintlich erst nach der Trennung der Eltern an die Stelle des Vaters. Und Pauls (Habibs) Entscheidung schließlich, sich nicht konfirmieren zu lassen, resultiert scheinbar allein aus dem Bestreben, authentisch $\mathrm{zu}$ handeln:

„Und als ich dann älter war, wollte meine Mutter auch, $\mathrm{daB}$ ich also konfirmiert werde, aber $\mathrm{hm}$, da war ich dann so im Alter von vierzehn Jahren etwa, das war dann ne Sache schon, wo ich eh, eh, mich dagegen sträubte. Ja also, ich hatte doch festgestellt, daß ich eh, eh, eh, das Christentum is mir irgendwie nie verinnerlicht worden irgendwie, ich hatte da nie irgendwie einen, hm, hm, ne feste Beziehung zu gehabt. Das war eben halt, das lief so nebenher so, aber das war eben halt keine Sache, die mich jetz irgendwie stark bewegte. Da hatt ich irgendwo ooch keine Bindung, keine Beziehung, gar nichts verband mich damit irgendwo; und eh eh zur Frage der Kon.., eh, eh, eh der Konfirmation sagt ich mir, das wäre unehrlich, jetzt etwas z.., eh, eh, zu bekräftigen oder hm, mich zu etwas zu bekennen, wo ich eigentlich gar nicht zu stehe. (...) Und des war irgendwo ne Sache, die eh, ph, und nur eben halt der 
Nachbarn wegen! Daß eben halt, daß es in der Nachbarschaft, eh, eh, nicht heißt: ,also der Sohn von da und da, der hat sich jetzt nich konfirmieren lassen!"“. Also mit dieser Schande konnt' ich also leben. Für meine Mutter war 's also, ooch doch irgendwo ' $n$ Treffer gewesen, unter der Gürtellinie....

Hier bekommt man den Eindruck eines auf Konformität bedachten Umfeldes, das den Verstoß gegen soziale Konventionen als „Schande“ betrachtet, und gegen das sich der auf Authentizität bedachte Sohn zur Wehr setzt.

Die komplette Rekonstruktion des biographischen Verlaufs zeigt jedoch einen völlig anderen Zusammenhang. Die Mutter ist es, die mit ihrem außerehelichen Verhältnis, der nichtlegalisierten Beziehung zu einem Angehörigen einer ethnischen Minderheit und der späten unehelichen Mutterschaft in verschiedener Hinsicht gegen die Sexualmoral eines bürgerlichen Settings verstößt. Ihr Ehemann jedenfalls drängt auf eine schnelle 'Bereinigung' der Situation, woraus man entnehmen kann, daß es sich nicht um ein Milieu handelt, in dem die „offene Ehe“ zum gelebten Programm geworden wäre.

Obwohl der Proband über die Reaktionen des sozialen Umfeldes, das sich ja nach der Trennung der Eltern nicht verändert hat, in seinem offenkundigen Versuch der Normalisierung nichts berichtet, kann man wohl davon ausgehen, daß es auf diese Ereignisse nicht gerade tolerant reagiert haben dürfte. Und auch ein elfjähriges, vom Stand der moralischen Entwicklung her an Konventionalität orientiertes Kind (vgl. Kohlberg 1971), wird vermutlich daran Anstoß genommen haben. Jedenfalls erwähnt der Proband, daß die Eltern zum Zeitpunkt der Trennung 27 Jahre ein Paar gewesen seien, was die Bedeutung der Trennung unterstreicht.

Das Problem der Normverletzung verschärft sich dadurch, daß die neue Beziehung der Mutter nicht legitimiert wird und überdies einige Jahre später wegen der sexuellen Eskapaden des Mannes wieder zerbricht. Wenn in dieser Zeit von "Schande“ die Rede gewesen sein wird, dürfte sich dies eher auf das Verhalten der Mutter als auf die nicht stattgefundene Konfirmation des Sohnes bezogen haben.

In diesem Versuch der Normalisierung wird aber gleichzeitig eine Transformation des Konfliktes auf eine andere Ebene erkennbar: Entweder die Mutter selbst oder - stellvertretend - der Sohn verschieben hier die Normverletzung von der Ebene der Sexualität auf die der Religion. Dabei handelt es sich um den ersten Schritt eines Transformationsprozesses, wie er für diesen Typus der Konversion charakteristisch ist.

An einer Stelle klingt diese Überlagerung des 'Problems Sexualität' durch das 'Problem Religion' im Text an. Im Rahmen der Ausführungen des Probanden über seine Konfirmation fällt die im Kontext einer Rede über Authentizität und Ehrlichkeit ungewöhnlich scharfe und deutlich sexuell konnotierte Äußerung auf, die Weigerung, sich konfirmieren zu lassen, sei für die Mutter „ein Treffer unter der Gürtellinie“ gewesen. Diese ÄuBerung transportiert - sparsam interpretiert - in jedem Fall eine aggressive Haltung der Mutter gegenüber, denn ein "Treffer unter der Gürtellinie“ ist kein zufälliges Resultat einer Handlung, sondern Folge eines bewußt und gezielt ausgeführten Schlages. Die symbolische Bedeutung dieses Ausdrucks knüpft zudem an die Plazierung solcher Schläge an: sie zielen auf den Intimbereich, stellen bloß und beschämen.

Insofern kann auch die sexualisierte Ausdrucksform als Beleg für die Lesart gelten, daß hier ein TransformationsprozeB stattgefunden hat: Die Abkehr von der Religion der Mutter und der damit verbundene Verstoß gegen die Konvention überlagert die Normverletzung der Mutter, verweist aber noch in der Sprachform auf das, wogegen die Aggression sich richtet: auf ihre Sexualität.

In diese Interpretation fügt sich noch ein weiterer Baustein: den Halbbruder, das gemeinsame Kind der Mutter und ihres Geliebten und damit das Produkt ihrer Sexualität, haßt Habib Bode als Jugendlicher. Als Erwachsener dagegen versucht er ihn, der ja eigentlich gebürtiger Muslim ist, vom Islam zu überzeugen.

\subsubsection{Habitualisierung einer religiös begründeten Moral}

Wie aus der biographischen Rekonstruktion ersichtlich wurde, vollzieht sich die Abwendung von der Religion der Mutter durch die Übernahme der Religion, der der afrikanische Stiefvater lediglich formal angehört. Es handelt sich also um keinen bloßen Vorgang der Identifikation, denn mit dem Tunesier teilt Paul zunächst einmal die säkulare Haltung: liegt diesem doch nichts ferner als das Einhalten der islamischen Gebote. Indem sich die formale Angleichung an die Religion des Afrikaners mit einer Überbietung verbindet - indem Paul als Habib zum besseren Muslim wird - wird gleichzeitig eine grundlegende Differenz zum Ausdruck gebracht. Auch diese artikuliert sich besonders auf 
dem Gebiet der Sexualität. Während der Stiefvater in 'ehebrecherischer' Beziehung zu seiner Mutter lebt, strebt Habib als junger Mann in Tunesien eine islamische Heirat mit einem Mädchen an, mit dem er noch kein Wort gewechselt hat. Und auch die Art, wie er schließlich in Deutschland die Ehe mit einer Kurdin eingeht, zeigt noch etwas von diesem Kontrastprogramm: eingeschworen auf die Perspektive, eine ausländische Muslime heiraten zu wollen, bittet er die Kurdin bereits bei der ersten Begegnung bei Freunden, seine Frau zu werden. So als solle die Kontingenz einer Liebesgeschichte von Anfang an ausgeschaltet werden.

Als Fazit dieser Interpretation ist festzuhalten, daß die Funktion der Konversion zum Islam, in diesem Fall der formalen Übernahme und faktischen Überbietung der Religion des afrikanischen Stiefvaters, in der Bewältigung der Normverletzung der Mutter liegt. Der Tunesier, der den Islam in den Horizont des Probanden einführt, liefert für diesen Prozeß lediglich das Material.

In die Form dieser Bewältigung ist die Kritik gleichzeitig eingeschmolzen. Charakteristisch dafür ist, daß das Problem des Verstoßes gegen Sexualnormen auf die religiöse Ebene verschoben und dadurch normativ zugespitzt, empirisch aber entschärft wird. Durch die Art der Aneignung des Islams wird eine religiös begründete Moral nicht nur abstrakt übernommen, sondern grundlegend habitualisiert. Von der Mutter als konkreter Person ist der Konflikt damit jedoch abgelöst.

Aufschlußreich ist in diesem Zusammenhang die Antwort, die der Proband am Ende des Interviews auf die Frage gibt, was für ihn „Religion“ sei:

\begin{abstract}
„Hm, nja, wie will man das jetz entwickeln. - Mhm, vielleicht so: Religion ist der Versuch des Menschen, sein Leben oder sein, sein, seine Taten und sein Handeln auf eine moralische Grundlage zu stellen. Und insofern akzeptier ich eigentlich auch alle anderen Religionen, ob das Christentum is oder Judentum oder auch Buddhismus, Hinduismus, Shintoismus und was weiß ich, was es alles gibt, denn jede Religion versucht ja eigentlich des Gleiche oder oder ja, stellt an seine Gläubigen die gleiche Forderung: nämlich ein Leben zu führen, was also von, von der Ethik, von Normen und von, von Moral bestimmt ist. Nehm wer mal die zehn Gebote. Die gibt es eigentlich in so ziemlich jeder Religion - in leicht abgeänderter Form! Oder nich als zehn Gebote definiert. Aber auch im Islam gilt: „Du solist nich töten, du sollst nich lügen, du sollst nich ehebrechen!" und dergleichen".
\end{abstract}

Die Gleichsetzung von Religion und Moral geht hier sogar so weit, daß darüber selbst die Sonderstellung, die im Islam den drei Buchreligionen zukommt, aufgegeben wird.
Eine letzte Bemerkung gilt der Frage nach funktionalen Äquivalenten: Welche im Rahmen seines Handlungsspielraumes realistischen Alternativen hätten sich dem Kind geboten, mit dem Normverstoß der Mutter umzugehen, immer vorausgesetzt, daß es ihr Verhalten als Normverletzung erfährt, was aufgrund der Normalisierungsbemühungen während des Interviews anzunehmen, was vom Kontext her plausibel und vom Entwicklungsstand eines Elfjährigen her erwartbar ist? Eine mögliche Alternative wäre sicher der Ausweg in ,abweichendes Verhalten" - Aufsässigkeit in der Schule oder gegenüber der Mutter - gewesen. Diese Variante hätte den Konflikt gegenüber der Mutter expliziter gemacht, wäre allerdings auch mit hohen Kosten verbunden gewesen: sie hätte den Probanden in verschiedener Weise isoliert.

Die Transformation in den religiösen Bereich ist im Vergleich dazu unmittelbar weniger konfliktträchtig, von ihrem latenten Sinngehalt her jedoch um so gravierender hinsichtlich der Bewertung der Mutter. Nicht zufällig kommentiert die Großmutter die Konversion des Enkels so, daß ihre Tochter dabei ihren Sohn verloren habe. Und ebensowenig zufällig ist es, daß der zweite, dunkelhäutige, vom Vater adoptierte und als Muslim erzogene Sohn, von der Mutter nach der Trennung von diesem Mann formal wieder zum „Christen“ gemacht wird und seinerseits die Spuren des afrikanischen Teils seiner Herkunft auszulöschen versucht.

\subsection{Fall II: Sexuelle Normverletzung und symbolische Externalisierung}

\subsubsection{Biographische Skizze}

Brigitte Haltun wächst in den 50er Jahren in einer kommunistischen Familie in Westdeutschland auf, deren Alltag charakterisiert ist durch exzessiven Alkoholkonsum und Gewalt zwischen den Eltern. Der Vater hat überdies jahrelang ständig außereheliche Beziehungen zu sehr jungen Frauen, bis er sich schließlich scheiden läßt und seine langjährige Geliebte heiratet, die jünger ist als seine Tochter Brigitte. Gleichzeitig wird die „Weiblichkeit" der Töchter tendenziell eliminiert: Sie werden eher „männlich“" sozialisiert: mit kurzen Haaren und einer auf Härte orientierten Erziehung. Brigitte wird als Kind mit einem männlichen Spitznamen gerufen. Beide Phänomene - die Reproduktion des Vater-Tochter-Verhältnisses in der Beziehung zur jungen Geliebten sowie die tendenzielle Umdefinition des Geschlechts der Töchter - deuten darauf, daß die Grenzziehungen im 
Verhältnis zwischen Vater und Töchtern eher prekär sind.

Die Probandin selbst verstrickt sich im Verlauf ihrer Biographie immer wieder in problematische Intimbeziehungen, in denen sie sich materiell und sexuell ausgenutzt fühlt. Früh bekommt sie ein uneheliches Kind und hat insgesamt vier Abtreibungen, bis sie sich schließlich sterilisieren läßt.

Im Alter von 30 Jahren hat Brigitte Haltun in privater wie auch beruflicher Hinsicht eine Geschichte des Scheiterns hinter sich: das angestrebte Abitur trotz mehrerer Anläufe nicht erreicht; verschiedene Berufsausbildungen abgebrochen; keine befriedigende Liebesbeziehung und darüber hinaus einen Sohn, bei dem sich ebenfalls massive Probleme abzuzeichnen beginnen. Ihr Engagement in der kommunistischen Partei ist zwar einerseits ein stabiles Element in ihrem Leben, aber auch hier erlebt sie Grenzüberschreitungen, die die Bekundung sozialistischer Solidarität unterminieren: als junge Genossin wird sie in ein Verfahren wegen sexuellen Mißbrauchs involviert, das männliche Jugendliche gegen 'altgediente' Genossen anstrengen, die in der kommunistischen $\mathrm{Ju}$ gendarbeit tätig sind.

Mit 30 Jahren sucht sie über ein Inserat einen Lehrer für Arabisch. Den Mann, der sich daraufhin meldet, einen 30 Jahre älteren Syrer, heiratet sie nach kurzer Zeit. Einige Zeit nach der Heirat tritt sie aus der DKP aus.

Als ihr Sohn 14 ist, kommt sie dahinter, daß er sich bereits seit längerer Zeit als Strichjunge verdingt. Sie schlägt ihm ein rigides Umerziehungsprogramm im Herkunftsland ihres Mannes vor, woraufhin er von zu Hause verschwindet. Als er nach längerer Zeit von der Polizei aufgegriffen wird, weigert sie sich, ihn wieder mit nach Hause zu nehmen. Seitdem ist der Kontakt zu ihm abgebrochen.

Nach einem Ägyptenaufenthalt beginnt Brigitte Haltun auch zu Hause in der Öffentlichkeit ein Kopftuch zu tragen, obwohl ihrem Mann daran nicht gelegen ist.

Im Verlauf der Ehe bricht sie sukzessive den Kontakt zu ihren alten Freunden ab und integriert sich in die Familie ihres Mannes. Zum ersten Mal gelingt es ihr, eine Ausbildung erfolgreich abzuschließen. Sie macht Pläne, das erstrebte Abitur doch noch nachzuholen und überlegt sogar, die Sterilisation rückgängig machen zu lassen.

\subsubsection{Symbolische Grenzziehung und externalisierte Attribution}

Was bereits die biographische Skizze andeutet, zeigt sich auch in Interviewausschnitten mit Brigitte Haltun. Zentrale Passagen kreisen - in einer überwiegend diskreditierenden Weise - um das Thema Sexualität.

Betrachtet man diese Schilderungen, so scheinen sich die sexuellen Beziehungen Brigitte Haltuns quasi selbstläufig zu entwickeln, jedoch nicht ohne anschließend ihre Verachtung nach sich zu ziehen. In diesem Zusammenhang erzählt sie folgende Episode. Es handelt sich um einen „Morgen danach":

„Dann hatt ich halt einen abgeschleppt, dann wurd ich wach und bekuck mir den, ich hätt kotzen können. Ich mein', der sah gar nicht schlecht aus oder wat, ja? Ich hab' nur gedacht: was machst $D u$ hier eigentlich? Und mußt auch noch bescheuert sein. So voller Stolz bezahlt frau ihre Rechnung selbst. Frau läßt sich doch nicht einladen. Das ist doch Schnee von gestern, ne. Wir ham unser eigenes Geld, und wenn hier jemand für beide bezahlt, dann bin ich dat, ne. Hab' ich gedacht: Du mußt doch so doof sein, ne. $\mathrm{Na}$ hab ich den rauskomplimentiert. Und dann war ich irgendwie, die Kinder warn im Kindergarten, ich hatte auch noch frei, kein Aas mehr da. Dann hab ich mich vor den Spiegel gestellt. Ich hätt kotzen können. Ich hab gedacht: Bist du bekloppt oder was? Wozu soll dat führen, ne? Alle paar Wochen schleppste da jemand ab, und dat waste suchst, findste sowieso nich. Also so, ja, jemand, mit dem de wirklich ne vertrauliche, vernünftige Beziehung ham kannst, wo dein Kind auch mit drin is."

Diese Szene $^{22}$ enthält verschiedene zentrale Motive: Hier geht es zum einen um eine klassische individualisierte Form der Selbstreflexion: das einsame Ich (,,kein Aas mehr da“) zieht sich vor dem Spiegel selbst zur Rechenschaft und fragt nach Ziel und Zweck des Handelns.

Gleichzeitig kommt in der Spiegelszene ein Verhältnis der Selbst-Verachtung zum Ausdruck (,Ich hätt kotzen können“). Diese Verachtung bezieht sich ausdrücklich nicht auf die Person des Mannes, sondern auf die Frau im Spiegel und wohl darüber hinaus auf das gesamte Setting.

Und schließlich - dies integriert die ersten beiden Momente - wird ein ökonomisches Kalkül artikuliert, in dem Sexualität Tauschobjekt ist für etwas anderes. Der Tausch, der stattgefunden hat, ist aber in doppelter Weise ungerecht. Dies zeigt sich in dem impliziten Verweis auf zwei Rollen, die ihn ausgewogener gestaltet hätten: es ist die Rolle der

${ }^{22}$ Genaueres zu diesem Fall findet sich in Wohlrab-Sahr 1995. 
Ehefrau, die als Gegenwert für die gewährte Sexualität die Sicherheit des Familienlebens erhielte (,ne vertrauliche, vernünftige Beziehung, ... wo dein Kind auch mit drin ist"), und es ist die Rolle der Geliebten, die sich vom Liebhaber zumindest „einladen" ließe. Beides ist hier nicht gegeben.

So verkehrt sich die aktive Rolle - die Probandin war es ja, die den Mann ,abgeschleppt“ und darüber hinaus die Zeche bezahlt hat - unter der Hand in ihr Gegenteil. Trotz der Auflösung der geschlechtstypischen Muster auf der Handlungsebene ist es letztlich gerade der Verstoß gegen eine geschlechtstypisch ausformulierte Sexualmoral, die die Preisgabe der Sexualität nur gegen einen angemessenen Gegenwert erlaubt, der zur Selbstverachtung führt. So wird die größere Freizügigkeit auf der Handlungsebene konterkariert durch eine in die Bewertung der Handlung eingewobene Ökonomie der Sexualität, in der geschlechtstypische Muster nach wie vor wirksam sind. ${ }^{23}$

Das innere Selbstgespräch vor dem Spiegel endet damit, daß Brigitte Haltun sich ihre langen Haare radikal abschneidet. Damit rekurriert sie auf ein Symbol, das die Episode implizit einreiht in eine Geschichte weiblicher Ent-Ehrung, ein Symbol allerdings, das kulturell nicht mehr verbindlich ist. Ihre Mitbewohner jedenfalls finden es lediglich „schade“, daß die langen Haare ab sind.

Im Verlauf des Interviews kommt Brigitte Haltun später noch einmal auf diese Episode zu sprechen. Sie schildert mehrere Situationen, in denen ihr deutlich geworden sei, wie ihre Haare oder die anderer Frauen Männer 'zum Träumen verführten', ihnen gewissermaßen als illegitime Anleihen für erotische Phantasien dienten. Diese Möglichkeit will sie heute ausschließen. Aus ihrer heutigen Sicht als Muslime erklärt dieses Eigenleben der Haare auch, warum sie sich früher immer wieder in Affären verstrickt hat: „Wenn du mit offenen Haaren rumläufst", resümiert sie, „musses sein". So interpretiert sie auch nachträglich das $\mathrm{Ab}$ schneiden der Haare als Stoppen dieser Dynamik. Heute dagegen sei dies nicht mehr nötig, da sie ihr Haar mit dem Kopftuch verhülle. Hier taucht also die oben geschilderte Episode in einem neuen Rahmen wieder auf.

23 Auch an anderen Stellen im Interview reproduziert sich diese ökonomische Perspektive auf Sexualität. So kommentiert Brigitte Haltun das freizügige sexuelle Verhalten einer Freundin so, daß diese allgemein als „die billigste Hure von St. Pauli“" angesehen werde, bei der man nicht nur nächtigen könne, sondern morgens auch noch ein Frühstück erhalte.
Diese neue Interpretation enthält nun ein Attributionsschema, das von der oben geschilderten Episode klar verschieden ist - ein zentrales Merkmal von Konversionen (s. Snow/Machalek 1984; Sprondel 1985). In der ersten Episode war mit der Erfahrung des 'ungleichen Tausches' Selbstverachtung verbunden, die erfahrene Entwertung wurde trotz der Verweise auf Geschlechtsidentität letztlich individuell zugerechnet (,Was machst du hier eigentlich?"). Hier zeigt sich nun eine andere entindividualisierte - Form der Attribution, die auch eingebettet ist in eine andere Form der Problemlösung.

Durch die religiös fundierte Interpretation, ihr offen getragenes langes Haar sei als Sexsymbol Ursache der übereilt zustande kommenden Affären, wird das Problem von der persönlichen Ebene auf eine allgemeine Ebene geschlechtstypischer Merkmale transformiert: Nicht sie persönlich braucht die Verachtung zu treffen, denn dem Frauenhaar generell haftet diese symbolische Bedeutung an, die es entsprechend zu berücksichtigen gilt. Auch hier findet also eine symbolische Transformation statt: Die symbolische Ordnung, auf die Brigitte Haltun hier rekurriert, hilft die alten Erfahrungen neu zu interpretieren und entlastet gerade von individualisierten Zurechnungen. Zudem wird durch den Bezug auf ein Familien- und Gesellschaftsmodell, nach dem Sexualität an Ehe gebunden und der Ehemann zur Versorgung verpflichtet ist, die Möglichkeit sexueller 'Beraubung' ohne Gegenleistung ausgeschlossen.

Die hier gefundene Lösung - die Übernahme der islamischen Kleidung und die Heirat des muslimischen Mannes - hat aber noch einen weiteren Bezug auf ein Problem der Biographie: auf das immer wieder auftauchende Problem verfließender Grenzen: im Verhältnis zum Vater, in der kommunistischen Partei, in der Steuerung der eigenen Sexualität. Solche Grenzen wurden in der Vergangenheit durch radikale Schnitte nachträglich errichtet, da sie in der Handlungssituation selbst nicht aufrechterhalten werden konnten. Auch dies zeigt die Episode vor dem Spiegel, die mit dem Abschneiden der Haare endet.

Dieses Problem wird im Zug der Konversion gleichermaßen 'präsentiert' wie transformiert: so verweist die Eheschließung mit dem sehr viel älteren muslimischen Mann zurück auf das Problem prekärer Grenzen zwischen Tochter und Vater. Und die islamische Kleidung dokumentiert unübersehbar eine Grenzziehung nach außen und entlastet dadurch gleichzeitig davon, in der Situation selbst Grenzen definieren zu müssen. 


\subsection{Fall III: Sexuelle Entwertung und personifizierte Ehre}

\subsubsection{Biographische Skizze}

Peter Manski stammt aus einer Arbeiterfamilie. Als Kind konvertiert sein Vater auf Betreiben der Großmutter plötzlich zum Katholizismus und bewirkt auch die Konversion der Söhne. Die Mutter wird in diesen Konversionsvorgang nicht einbezogen.

Als Peter zwölf ist, lassen sich seine Eltern scheiden, nachdem der Vater in betrunkenem Zustand die Mutter immer wieder verprügelt hat. Im Alter von 16 Jahren setzt ihn seine Mutter vor die Tür, der Kontakt zu ihr bricht daraufhin ab. Die Großmutter bleibt eine zentrale Bezugsperson, Peter Manski verbringt viel Zeit bei ihr und überlegt sogar als Erwachsener, mit ihr zusammenzuziehen.

Während Peter als Kind von der Symbolik des Katholizismus fasziniert ist, verliert er als Jugendlicher den Kontakt zur Kirche und tritt zu Beginn der Berufstätigkeit wegen der Kirchensteuer aus.

Er macht zwei handwerkliche Ausbildungen, mit denen sich jedoch kaum Geld verdienen läßt und pachtet schließlich ein Lokal. Dort lernt er eine Frau kennen, die bald ein Kind von ihm erwartet. Die beiden ziehen zusammen und bekommen ein weiteres Kind. Peter Manski ist allein erwerbstätig und wegen der Arbeit im Lokal wenig zu Hause. Er fängt an zu trinken, muß schließlich das Lokal aufgeben und wechselt daraufhin zum Straßenbau. Obwohl das seinen Alltag stabilisiert, erlebt er den beruflichen Statusverlust als Scheitern einmal vorhandener Möglichkeiten. Dies verstärkt sich dadurch, daß er in seiner Familie den Status des Alleinernährers verliert, weil sein Lohn für den Unterhalt der Familie nun nicht mehr ausreicht. Schließlich kommt es in seiner Partnerschaft zu einer massiven Krise. Seine Lebensgefährtin hat einen Liebhaber und bleibt häufiger nachts weg. Als sie eines Morgens nach Hause kommt, verliert Peter Manski die Beherrschung und schlägt sie. Erschrocken über diese Dynamik sucht er sich innerhalb weniger Tage eine eigene Wohnung.

In der Zeit nach der Trennung kommt er über türkische Kollegen in Berührung mit dem Islam. Er ist vor allem angetan von der Intensität der Glaubenspraxis, die er in der Moschee erfährt, aber auch von der religiösen Verankerung der Lebensführung. So wendet er sich schließlich - noch ohne allzu große Verbindlichkeit - selbst dem Islam zu. Dies ändert sich, als er bei türkischen Kollegen eine Türkin kennen- und liebenlernt, die selbst in Scheidung lebt.

Über den Kontakt zu dieser Frau intensiviert sich Peter Manskis Einbindung in den Islam. Im selben Maße gerät er jedoch in Konflikt mit den alten Bezugsgruppen. Die Tatsache, daß er keinen Alkohol mehr trinkt, entfremdet ihn von den alten Freunden. Und als er die Türkin heiratet, führt dies zum weitgehenden Kontaktabbruch mit seiner Familie.

\subsubsection{Die religiöse Dimension des Ehrgefühls}

Wie bereits aus dieser biographischen Skizze deutlich wurde, vermittelt sich die religiöse Einbindung Peter Manskis stets über enge Bezugspersonen. Religiöse Zugehörigkeit bedeutet - neben den symbolischen Qualitäten - immer auch persönliche Bindung und Anerkennung. Als die protestantische Mutter seine Kinderzeit mit dem Hinauswurf beendet, bietet die katholische Großmutter eine Möglichkeit, diese zu verlängern; und in einer Situation, in der es ihm an Anerkennung und $\mathrm{Zu}$ gehörigkeit in verschiedener Hinsicht mangelt, nehmen ihn die türkischen Kollegen in die eigene Gruppe auf.

Das Problem mangelnder Anerkennung spitzt sich zu im Konflikt mit der Lebensgefährting, der am Ende einer Kette von Statusverlusten steht: er verliert seine berufliche Selbständigkeit zugunsten einer Tätigkeit mit geringem Prestige und damit auch seinen Status als Alleinernährer in der Familie, ohne daß es gelingt, diese Situation in ein partnerschaftliches Arrangement einmünden zu lassen; und er wird überdies als Sexualpartner entwertet, indem seine Lebensgefährtin sich vor seinen Augen einem anderen Mann zuwendet. Diese dreifache Entwertung kulminiert in der Gewalt gegenüber seiner Lebensgefährtin und endet darin, daß er beschämt das Feld räumt.

Die neue Beziehung zu der türkischen Muslime, die von den Arbeitskollegen in gewisser Weise 'vermittelt' wird, ist dazu in verschiedener Hinsicht ein Kontrast- und Reparaturprogramm. In der folgenden Passage schildert Peter Manski die erste Begegnung:

„.... waren ins Gespräch gekommen, (...) und dann ist, wie soll man sagen, das ist alles nicht so frei, so frei wie unter uns Deutschen, ne, is eben, dat Ehr, dieses Ehrgefühl, man hat da als Mensch, find ik ooch, jeder hat ne andere Einstellung, aber ik hab eben bei ihr ooch gleich gemerkt, nicht gleich: du lernst se jetzt kennen und dann nimmste se mit nach Hause und dann geht se mit dir ins Bett und dann ist fertig oder so. Man hatte, ik hatte vor ihr, ich habe irgendwie, kann man sagen: 'Liebe auf den ersten Blick', aber ich hatte auch so $n$, so $n$ Ehrgefühl, ja, oder auch $R e$ - 
spekt kann man sagen. Erstemal vor ner Frau, daß ich $R e$ spekt hatte, ne.

(...) Ja, war alles sehr dramatisch. (...) Hab immer gedacht, jetzt hast de ooch alles so was miterlebt und mitangesehen bei deine Eltern, bei dir selber und .. ja dann hatten wir das ganze Theater (...) mit der Scheidung und, war ne schlimme Zeit. /mhm/ Und (...) jedenfalls (...) wie wir zusammen gekommen sind, da hab ik gesagt Mensch, wa, wat, lieber Gott, du hast, irgendwie hast de mir doch geholfen, mal gibst de mir schlechtes, mal gibst du mir böses, da hat mich dann, dat dat nich nur von mir alleine kam, weil ich auch so n Ehrgefühl vor ihr hatte, dacht ich, jetzt mußt du dich mal mehr um deine, um die islamische Sache kümmern."

Im Gegensatz zur Kette von Statusverlusten und Entwertungserfahrungen im Verlauf der Biographie vermittelt sich hier die eigene Aufwertung über den ,Wert' der Frau, eine Vorstellung, die im Begriff der „Ehre" implizit enthalten ist. Die Beziehung zur neuen Frau ist einerseits individualisiert - „Liebe auf den ersten Blick“ - verbindet sich aber gleichzeitig mit einem sozialen Gefühl, einem „Ehrgefühl“" oder „Respekt“, der gewissermaßen der sozialen Kategorie „Frau“ gilt, die ihm in dieser Person begegnet. Dabei geht es nicht primär um äußere Merkmale wie Kleidung etc., sondern um einen eher habituell wahrgenommenen Unterschied und um das Verhältnis, das sich im Kontakt der beiden herstellt: bei ihm stellt sich ihr gegenüber ein Ehrgefühl her, es etabliert sich ein Geschlechterverhältnis, das sich von seinen bisherigen Beziehungen zu Frauen unterscheidet.

Diesen Unterschied interpretiert Peter Manski als Resultat anderer kultureller Regeln - es sei nicht so „frei“" wie unter Deutschen - die er sich zu eigen macht, und über die sich letztlich das Ehrgefühl vermittelt.

In der Thematisierung des Ehrgefühls gegenüber der Türkin 'als Frau' spielt auch die Anerkennung einer Geschlechterdifferenz eine Rolle. Im Unterschied dazu handelt es sich bei dem typisierten One-night-stand um eine Situation, die Mann und Frau gleichermaßen als unverbindlich definieren (, $d u$ lernst se jetzt kennen und dann nimmste se mit nach Hause und dann geht se mit dir ins Bett und dann ist fertig oder so"). Dagegen wird die Begegnung mit der neuen Frau durch andere Regeln gesteuert, die ihm die Anerkennung eines besonderen Verhaltens ihr gegenüber abverlangen.

Diese Differenz realisiert sich in der neuen Beziehung auf verschiedene Weise. Diese ist erkennbar Liebesbeziehung, entspricht aber gleichzeitig einer klassischen Rollenverteilung. Im Unterschied zu seiner gescheiterten Partnerschaft wird die Verbindung hier von Anfang an institutionalisiert. Peter Manski ist wieder Alleinverdiener, seine Frau ist Hausfrau, und ihre Ansprüche sind bescheiden genug, um dieses Setting nicht infrage zu stellen. Der Islam - von den beiden als eine Religion der Bescheidenheit interpretiert - liefert dazu das entsprechende religiöse Deutungsmuster. Gleichzeitig ist die türkische Frau Wächterin ehelicher Treue, indem sie es moniert, wenn deutsche Frauen ihrem Mann gegenüber zu vertraulich werden. Darüber bestätigt sie aber implizit auch seinen Status und die Differenz der Geschlechter: ist er doch in der Rolle dessen, der Offerten bekommt und nicht mehr in der Gefahr, seinerseits betrogen zu werden.

Das empfundene Ehrgefühl und die damit verbundene Aufwertung seiner männlichen Identität wiederum verschweißen Peter Manski mit der Religion. Denn weder die Begegnung mit der türkischen Frau noch das Ehrgefühl kann er sich individuell zuschreiben: „dat dat nich nur von mir alleine kam“", ist ihm klar. Damit bekommt das Gefühl in sich nahezu religiöse Qualität. In fast Durkheim'schem Sinne, wenn auch in scheinbar privaten Formen, verschmilzt hier Religion mit sozialem Sentiment (Durkheim 1981), mit „Ehrgefühl".

Auch hier wird in der, aus der christlichen Alltagsreligiosität stammenden Anrede Gottes (, lieber Gott") erkennbar, daß nicht der Islam als Glaubenskodex im Vordergrund steht, sondern als Religion, die mit „Ehre“, mit Moral verschmolzen ist. Diese Verschmelzung ist es dann letztlich auch, die die Konversion perfekt macht.

Im Interview wird verschiedentlich deutlich, in welchem $\mathrm{Ma \beta}$ es bei dieser Konversion um die Wiederherstellung des eigenen Wertes 'als Mann' geht. Eine zentrale Rolle in dem Gespräch, während dessen auch Peter Manskis Frau anwesend ist, nimmt die Geschichte seiner Beschneidung ein, die hier deutlich nicht primär als religiöse Vorschrift angesehen wird. In einer Passage hoher interaktiver Dichte schildern und kommentieren beide Ehepartner ausführlich die Vorbereitung und Durchführung der Beschneidung inklusive der damit verbundenen Gefühle von Angst und anschließendem Stolz. Deutlich wird dabei, daß es bei diesem Passageritus auch um ein Anerkennungsritual geht: um die Wiederherstellung der männlichen Geschlechtsidentität und der damit verbundenen Stellung im Ehesystem sowie um die Eingliederung in einen neuen sozialen Kontext. Die neue Peergroup der türkischen Kollegen ver- 
schafft dieser Handlung die nötige soziale Bestätigung: ein Initiationsritus auch in dieser Hinsicht.

Auch in diesem Fall verbindet sich also mit der Konversion eine symbolische Transformation, indem sich über die Frau, die mit dem Islam identifiziert wird, gleichzeitig die 'Reparatur' der männlichen Geschlechtsidentität vermittelt. Diese Verbindung kommt zum Ausdruck im sozialen Gefühl der „Ehre“ und wird symbolisch präsentiert im Ritus der Beschneidung: gleichermaßen religiös verankert, wie vom Sinngehalt auf Geschlechtsidentität zielend.

\subsection{Symbolische Transformationen: drei Varianten eines Typus}

In den skizzierten Fällen fungiert die Konversion zum Islam als Lösung für das Problem sozialer Entwertung, das aus der Erfahrung der Verletzung von Sexualnormen resultiert. Diese Normverletzung geht dabei von verschiedenen Personen aus, wird aber in ihrer Konsequenz von den Konvertiten selbst erfahren und von ihnen verarbeitet. Im Falle der beiden männlichen Konvertiten sind es signifikante weibliche Bezugspersonen - die Mutter oder die Lebensgefährtin - die sexuelle Normen verletzen: im einen Fall manifestiert sich dies in der außerehelichen Beziehung der Mutter zu einem Afrikaner; im anderen Fall in der sexuellen Untreue der Lebensgefährtin. Die weibliche Konvertitin verstößt selbst gegen sexuelle Normen, allerdings mag auch dies einen Hintergrund in einer prekären Grenzziehung zwischen Vater und Tochter haben.

In diesen Unterschieden zwischen männlichen und weiblichen Konvertiten reproduziert sich die Geschlechtstypik verletzter "Geschlechtsehre“, wie sie Sozialhistoriker beschreiben: „Geschlechtsehre" ist traditionell gebunden an das Verhalten der Frau. Diese schädigt mit einer sexuellen 'Verfehlung' nicht nur sich selbst, sondern auch den Mann und die ganze Familie.

Die Fälle von Konversion, um die es hier ging, lassen sich als Strategien der Reparatur verletzter "Geschlechtsehre" begreifen. Diese Strategien sind jeweils verschieden, vollziehen sich jedoch alle in Form einer symbolischen Transformation. Im Falle von Habib Bode findet die Auseinandersetzung mit der Normverletzung der Mutter einen komplexen Ausdruck in der konsequenten und dabei den Liebhaber der Mutter überbietenden Übernahme des Islams im Sinne einer Religion der Moral und in einer bis in körperliche Repräsenta- tion und die Gestaltung der eigenen Intimbeziehung hineinreichende Adaption dessen, was ihm eine konsequente - methodische - islamische Lebensführung zu sein scheint. Eine Religion der Moral auch, weil sie streng untersagt, worin die Normverletzung der Mutter bestand: den Ehebruch. Die Transformation dieser Auseinandersetzung in den religiösen Sinnbereich entlastet aber gleichzeitig von der direkten persönlichen Auseinandersetzung mit der Mutter. Der Konflikt muß dadurch, daß er eine religiöse Codierung erfahren hat, als Mutter-Sohn-Konflikt nicht mehr ausgetragen werden.

Im Fall von Peter Manski erfolgt die Transformation auf anderem Wege. Hier vermittelt sich nicht nur die sexuelle Normverletzung, sondern auch die Reparatur der damit verbundenen Entwertung über eine andere Person. Ist es das Verhalten der Lebensgefährtin, das ihm seinen Statusverlust ,als Mann" in beschämender Weise vor Augen führt, so ist es ebenfalls wieder eine Frau, die ihm die gegenteilige Erfahrung von Aufwertung vermittelt, wie sie im Begriff des „Ehrgefühls“ zum Ausdruck kommt. In diesem Fall impliziert die Konversion keine methodische Lebensführung, sondern das Gefühl neu gewonnener Wertschätzung, in dem Religion und ein spezifisches Geschlechterarrangement untrennbar verschmelzen und sich gegenseitig stützen.

Im Fall der Brigitte Haltun schließlich manifestiert sich die Normverletzung im eigenen sexuellen Verhalten, in der fehlenden Selbstkontrolle und Fähigkeit zur Grenzziehung, und nicht zuletzt darin, daß sie aufgrund des fehlenden 'Gegenwerts' zur Sexualität offenkundig ihren Wert als Frau gemindert sieht. Die Problematik der Entwertungserfahrung besteht hier darin, daß größere sexuelle Freiheitsspielräume konterkariert werden durch die gleichzeitige - und zwar eindeutig gegen die Frau gerichtete - Diskreditierung dieses Verhaltens. Trotz neuer Handlungsspielräume zeigt sich auch hier das Nachwirken sozialer Normen im Bereich von Sexualität und Geschlechterverhältnis, deren Verletzung gleichzeitig das Selbstwertgefühl verletzt. Um es in Abwandlung der Terminologie Simmels (1908: 405) zu formulieren - es zeigt sich, wie die Verletzung der 'sozialen Pflicht' zum 'individuellen Unheil' wird.

Die symbolische Transformation besteht hier in einem Prozeß der Externalisierung der Verantwortungszuschreibung und der Etablierung äußerer Grenzen, die an die Stelle selbstgesteuerter Grenzziehungen treten. Die äußere Erscheinung - die 
langen Haare - werden als Ursache der problematischen Sexualität identifiziert und mit einer entsprechenden Maßnahme - der Verhüllung - kuriert. Auch hier geht es nicht um eine methodische Lebensführung, sondern um eine symbolische Grenzziehung, in der sich ein Rahmenwechsel, die Verlagerung der Verantwortungszuschreibung von der Person weg hin auf ihr Äußeres manifestiert.

\section{Der Islam als Religion der Moral - Konversion als Implementation von Ehre}

In den Fällen, die hier rekonstruiert wurden, spielen implizite oder explizite Vorstellungen von Ehre und Schande, von moralischer und unmoralischer Lebensführung eine zentrale Rolle, und der Bereich der Sexualität ist dabei zentral. Bei den für die Konversionen charakteristischen Transformationsprozessen ging es um die symbolische Transformation der Erfahrung verletzter Sexualnormen in den religiösen Bereich hinein, um Prozesse der Sinnverschiebung und neuen Rahmung.

Nun scheinen sich Soziologen und Sozialhistoriker darin einig, daß "Ehre" in Gesellschaften unseres Typs ein ebenso veraltetes Konzept ist wie etwa „Jungfräulichkeit" (Berger 1970) und als Orientierungswert lange ausgedient hat. "Individualisiert“ - so Ute Frevert (1995: 222) - „auf die einzelne Person bezogen und abgekoppelt von normativ besetzten, hierarchisch strukturierten Gruppenund Geschlechteridentitäten" habe "das Konzept der Ehre seine vergesellschaftende Kraft zunehmend eingebüßt und sich selber überflüssig gemacht".

Peter L. Berger (1970) hat darauf hingewiesen, daß das Konzept der Ehre (honor) abgelöst worden sei durch das der Menschenwürde (dignity). Während Ehre als das zentrale Vergesellschaftungsprinzip einer hierarchischen, vor allem ständisch gegliederten Gesellschaft eng an institutionelle Rollen gekoppelt gewesen sei, gehöre zum Konzept der Menschenwürde essentiell gerade die Unabhängigkeit von institutionellen Rollen, sie beziehe sich auf das Menschsein an sich, losgelöst von askriptiven Merkmalen oder Statuspositionen.

Berger analysiert die Ablösung von Ehre als einen Proze $\beta$ der Individualisierung und De-Institutionalisierung und vermutet, daß eine 'Rückkehr $\mathrm{zu}$ den Institutionen' auch eine 'Rückkehr zur Ehre', wenn auch in einem veränderten Sinne, zur Folge haben werde.

Der gesellschaftliche Prozeß der Individualisierung impliziert nicht zuletzt auch eine veränderte
Zurechnung des Handelns. Damit erodieren langfristig auch die Grundlagen der spezifischen Ökonomie, die dem Denken in Kategorien der Ehre inhärent war und die den Wert einer Person danach bemaß, inwieweit sie mit den Regeln für eine bestimmte soziale Kategorie von Personen konform ging. Dies zeigt sich in besonderer Weise am Beispiel der Geschlechtsehre. ${ }^{24}$

Mit der Erosion von Ehre als Vergesellschaftungsprinzip verschwinden jedoch nicht per se die Erfahrungen sozialer Entwertung (vgl. Neckel 1991), die vormals in Kategorien der Ent-Ehrung gefaßt wurden. Es verschwinden zunächst nur die damit verbundenen kulturellen Institutionen: verbindliche und legitime Ausdrucksformen, aber auch vorgeschriebene Formen und Möglichkeiten der Reparatur.

Die Frage ist, was an deren Stelle tritt. Bleiben mit der Erosion von Ehre allein beschämende Erfahrungen individueller Unzulänglichkeit und entsprechend individualisierte Formen der Verarbeitung?

An dieser Stelle kommt nun die Konversion zum Islam ins Spiel. Die Konversion zum Islam präsentiert - das hat die Analyse gezeigt - eine spezifische Lösung für Erfahrungen sozialer Entwertung im Kontext sexueller Normverletzung. Diese Lösung besteht im Beerben eines kulturellen und religiösen Systems, das Ehrkodizes noch zur Verfügung stellt. Die Symbolik der Ehre und die damit verbundenen Verhaltenserwartungen werden über den Islam gewissermaßen von außen in ein kulturelles System implementiert, zu dessen sozial verbindlichem Inventar eine solche Symbolik nicht mehr gehört, das aber dennoch Phänomene sozialer Entwertung produziert.

Innerhalb des eigenen kulturellen Kontexts wird also auf ein anderes kulturelles Modell Bezug genommen, und es wird so im Bezug auf eine andere kulturelle Gegenwart die eigene kulturelle Vergangenheit wiederbelebt. Das Mittel dafür ist die Konversion zum Islam und - häufig in Verbindung damit - die Eheschließung mit einem Partner, der dieser Religion angehört und dem die Aufgabe zukommt, den religiös-kulturellen Habitus zu repräsentieren.

${ }^{24}$ Ute Frevert (1995) weist darauf hin, daß deren Bedeutung mit der Durchsetzung der Liebesheirat zunächst einmal anstieg und sich auf Keuschheit bzw. sexuelle Treue verengte, in der nun der zentrale Ausdruck von Liebe gesehen wurde. Längerfristig allerdings hat der Individualisierungsproze $\beta$ dem Konzept der Geschlechtsehre dann doch den Boden entzogen. 
Bei den Konversionen des hier skizzierten Typs findet sich - trotz mancher Unterschiede in der äuBeren Erscheinungsform und in den vertretenen Positionen - doch ein gemeinsames Muster, das den Islam in spezifischer Weise vereindeutigt zu einer Religion der Moral, das die Erfahrungen verletzter Sexualnorm aufgreift und ihnen eine religiös-symbolische Ausdrucksform gibt. Es ist allerdings auch deutlich, $\mathrm{da} ß$ es sich bei dieser Vereindeutigung um einen Konstruktionsvorgang handelt, und nicht um einen essentialistisch zu definierenden Grundzug des Islams.

Was im Zuge dieser Konversionen geschieht, kann man als kulturelle Legierung bezeichnen: die Symbolisierung eines Problems, das im Kontext einer Kultur entstanden ist, mit bestimmten Ausdrucksformen einer anderen. Vielleicht ist der Islam als Mittel dafür aber auch in spezifischer Weise geeignet, weil sich in ihm - so wie er europäischen Konvertiten begegnet - kultureller Partikularismus und religiöser Universalismus in besonderer Weise verbinden: die Gleichheit der Menschen vor Gott und der Partikularismus einer geschlechtstypisch ausformulierten ,islamischen Lebensführung“" (vgl. Schiffauer 1983: 68). ${ }^{25}$

\section{Literatur}

Al-Azmeh, A., 1993: Islams and Modernities, London: Verso.

Asad, M., 1992: Der Weg nach Mekka. Reporter, Diplomat, islamischer Gelehrter: Das Abenteuer eines Lebens. Hamburg: Luchterhand.

Berger, P.L., 1970: On the Obsolescence of the Concept of Honor. Archives europeenes de sociologie 11:339-346.

Berger, P.L., 1988: Zur Dialektik von Religion und Gesellschaft. Elemente einer soziologischen Theorie, Frankfurt a.M.: Fischer (im Original 1967).

Berger, P.L./Kellner, H., 1965: Die Ehe und die Konstruktion der Wirklichkeit. Soziale Welt 16: 220-235.

Colpe, C., 1989: Kopftuch und Schleier: Was verbergen sie, was sprechen sie aus? S. 105-125 in: ders.: Problem Islam, Frankfurt a.M.: Athenäum.

${ }^{25}$ Man kann sicher fragen, ob es nicht auch in der eigenen Kultur Formen einer solchen hochintegrierten ordnungsstiftenden ,religiösen Lebensführung“ gibt. Für die skizzierten Fälle sind zumindest zwei funktionale Äquivalente vorstellbar: die katholische Kirche und eine auf Differenz setzende Fraktion des Feminismus. Im Katholizismus allerdings verflüchtigt sich die Verbindung von konfessionell geprägter Religion und einer spezifischen Form der Lebensführung zunehmend (s. Tyrell 1993). Zwischen einem auf Differenz setzenden Feminismus und dem Islam dagegen scheinen die Übergänge innerhalb des vorliegenden Samples oft fließend.
Durkheim, E., 1981: Die elementaren Formen des religiösen Lebens. Frankfurt am Main: Suhrkamp.

Frevert, U., 1995: „Mann und Weib, und Weib und Mann". Geschlechter-Differenzen in der Moderne. München: Beck.

Garaudy, R., 1992: Verheißung Islam. München: SKD Bavaria.

Glaser, B.G./Strauss, A.L., 1967: The Discovery of Grounded Theory. Strategies for Qualitative Research. Chicago: Aldine.

Hofmann, M., ${ }^{21993: ~ D e r ~ I s l a m ~ a l s ~ A l t e r n a t i v e . ~ M u ̈ n c h e n: ~}$ Diederichs.

Ingram, L. C., 1989: Evangelism as Frame Intrusion: Observations on Witnessing in Public Places. Journal for the Scientific Study of Religion 28: 17-26.

Jones, K., 1977: Some Epistemological Considerations of Paradigm Shifts. The Sociological Review 25: 253-271.

Jones, K., 1978: Paradigm Shifts and Identity Theory: Alternation as a Form of Identity Management. S. 5982 in: Mol, H. (Hrsg.), Identity and Religion. International, Cross-Cultural Approaches. Beverly Hills.

Kilbourne, B./Richardson, J.T., 1988: Paradigm Conflict, Types of Conversion, and Conversion Theories. Sociological Analysis 50: 1-21.

Kohlberg, L., 1971: From Is to Ought. S. 151-236 in: Mishel, Th. (Hrsg.): Cognitive Development and Epistemology, New York.

Krech, V., 1995: Was ist religiöse Bekehrung? Ein Streifzug durch zehn Jahre soziologischer Konversionsforschung. Handlung, Kultur, Interpretation 4, 6: 131199.

Kuhn, Th.S., 1976²: Die Struktur wissenschaftlicher Revolutionen. Frankfurt a.M.: Suhrkamp.

Kuhn, Th. S., 1977: Neue Überlegungen zum Begriff des Paradigma. S. 389-429 in: ders., Die Entstehung des Neuen. Studien zur Struktur der Wissenschaftsgeschichte, Frankfurt a.M.: Suhrkamp.

Langer, S., 1984: Philosophie auf neuem Wege. Das Symbol im Denken, im Ritus und in der Kunst. Frankfurt a.M.: Fischer.

Leggewie, C., 1993: Alhambra - Der Islam im Westen. Reinbek: Rowohlt.

Lofland, J./Stark, R., 1965: Becoming a World-Saver: A Theory of Conversion to a Deviant Perspective. American Sociological Review 30: 862-875.

Luckmann, Th., 1986: Grundformen der gesellschaftlichen Vermittlung des Wissens: Kommunikative Gattungen. S. 191-211 in: Neidhardt, F./Lepsius, M.R./ Weiss, J. (Hrsg.), Kultur und Gesellschaft, Sonderheft 27 der Kölner Zeitschrift für Soziologie und Sozialpsychologie, Opladen: Westdeutscher.

Luhmann, N., 1970: Funktionale Methode und Systemtheorie. S. 31-53 in: ders.: Soziologische Aufklärung. Aufsätze zur Theorie sozialer Systeme, Band 1, Opladen: Westdeutscher.

Mead, G.H., 1973: Geist, Identität, Geseilschaft. Frankfurt a.M.: Suhrkamp.

Merton, R.K., 1995: Soziologische Theorie und soziale Struktur. Berlin/New York: de Gruyter. 
Neckel, S., 1991: Status und Scham. Zur symbolischen Reproduktion sozialer Ungleichheit. Frankfurt a.M.: Campus.

Oevermann, U./Allert, T./Konau, E./Krambeck, J., 1979: „Die Methodologie einer „objektiven Hermeneutik" und ihre allgemeine forschungslogische Bedeutung in den Sozialwissenschaften“. S. 352-434 in: H.-G. Soeffner (Hrsg.): Interpretative Verfahren in den Sozial- und Textwissenschaften, Stuttgart: Metzler.

Poston, L., 1992: Islamic Da'wah in the West. Muslim Missionary Activity and the Dynamics of Conversion to Islam. New York/Oxford: Oxford University Press.

Richardson, J.T., 1978: Introduction. S. 5-9 in Richardson, R. (Hrsg.), Conversion Careers. In and Out of the New Religions. Beverly Hills/London: Sage.

Schiffauer, W., 1983: Die Gewalt der Ehre. Erklärungen zu einem türkisch-deutschen Sexualkonflikt. Frankfurt a.M.: Suhrkamp.

Schütze, F, 1976: Zur Hervorlockung und Analyse thematisch relevanter Geschichten im Rahmen soziologischer Feldforschung. S. 159-260 in: Arbeitsgruppe Bielefelder Soziologen (Hrsg.): Kommunikative Sozialforschung, München: Fink.

Schütze, F, 1983: Biographieforschung und narratives Interview. Neue Praxis 3: 283-293.

Schwabenthan, S., 1990: Islam. Warum immer mehr Deutsche zu Allah beten. marie claire 7: 8-16.

Simmel, G., ${ }^{6} 1983$ : Soziologie. Untersuchungen über die Formen der Vergesellschaftung. Berlin: Duncker \& Humblot.

Snow, D.A./Machalek, R., 1983: The Convert As A Social Type. S. 259-289 in: R. Collins (Hrsg.), Sociological Theory, Vol 1. San Francisco.

Snow, D.A./Machalek, R., 1984: The Sociology of Conversion. Annual Review of Sociology 10: 167-190.

Sprondel, W.R., 1985: Subjektives Erlebnis und das Institut der Konversion. S. 549-558 in: B. Lutz (Hrsg.), So- ziologie und gesellschaftliche Entwicklung. Verhandlungen des 22. Deutschen Soziologentages in Dortmund 1984, Frankfurt a.M./New York: Campus.

Stromberg, P., 1991: Symbols into Experience: A Case Study in the Generation of Commitment. Ethos 19: $102-126$.

Thömmes, J. 1993: Islamischer Fundamentalismus in Frankreich. Die ,affaire des foulards" 1989. S. 292-308 in: J. Bergmann/A. Hahn/Th. Luckmann (Hrsg.), Religion und Kultur. Sonderheft 33 der Kölner Zeitschrift für Soziologie und Sozialpsychologie. Opladen: Westdeutscher Verlag.

Tyrell, H., 1993: Katholizismus und Familie - Institutionalisierung und De-Institutionalisierung. S. 126-149 in: J. Bergmann/A. Hahn/Th. Luckmann (Hrsg.), Religion und Kultur. Sonderheft 33 der Kölner Zeitschrift für Soziologie und Sozialpsychologie. Opladen: Westdeutscher Verlag.

Ulmer, B., 1988: Konversionserzählungen als rekonstruktive Gattung. Erzählerische Mittel und Strategien bei der Rekonstruktion eines Bekehrungserlebnisses. Zeitschrift für Soziologie 17: 19-33.

Ulmer, B., 1990: Die autobiographische Plausibilität von Konversionserzählungen. S. 287-295 in: W. Sparn (Hrsg.): Wer schreibt meine Lebensgeschichte? Biographie, Autobiographie, Hagiographie und ihre Entstehungszusammenhänge. Gütersloh: Mohn.

Watson, H., 1994: Women and the Veil. Personal Responses to Global Process. S. 141-159 in: A.S. Ahmed/ H. Donnan (Hrsg.), Islam, Globalization and Postmodernity. London/ New York: Routledge.

Wohlrab-Sahr, M., 1995: Das Unbehagen im Körper und das Unbehagen in der Kultur. Überlegungen zum Fall einer Konversion zum Islam. S. 285-311 in: dies. (Hrsg.), Biographie und Religion. Zwischen Ritual und Selbstsuche, Frankfurt a.M.: Campus. 